\title{
Promising Approach to Inhibit E. coli FimH Adhesion by $C$-Linked Mannosides ${ }^{\dagger}$
}

\author{
Leila Mousavifar * and René Roy * \\ Department of Chemistry, Université du Québec à Montréal, P.O. Box 8888, Succ. Centre-Ville, \\ Montréal, QC H3C 3P8, Canada \\ * Correspondence: leilyanmousavifar@gmail.com (L.M.); roy.rene@uqam.ca (R.R.) \\ + Presented at the 2nd Molecules Medicinal Chemistry Symposium (MMCS): Facing Novel Challenges in \\ Drug Discovery, Barcelona, Spain, 15-17 May 2019.
}

Published: 6 August 2019

Keywords: carbohydrate; D-mannosides; uropathogenic infections; E. coli; lectin; FimH

Antagonists of the uropathogenic Escherichia coli type-1 fimbrial adhesin (FimH) are recognized as attractive alternatives for antibiotic therapies and prophylactic strategies against acute and recurrent bacterial infections.

In this study, $C$-linked $\alpha$-D-mannopyranosides possessing aromatic aglycons were investigated to fit within the hydrophobic pocket of the FimH Tyrosine gate (Tyr48-Tyr137). The results were summarized into a set of structure-activity relationships to be used toward FimH-targeted inhibitor design. Alkene linkers afforded improved affinity and inhibitory potential, because they could provide favorable binding interactions with hydrophobic side chains located in the middle of the tyrosine gate.

Of particular interest was a $C$-mannoside derivative, prepared by a Heck reaction between a family of aryl iodides and C-allyl $\alpha$-D-mannopyranoside. One of them, an ortho-substituted biphenyl aglycone, showed an affinity enhancement in the $\mathrm{nM}$ range. Docking of its high-resolution NMR solution structure to the FimH adhesin indicated that it could present its ortho-substituted phenyl ring directly in contact with isoleucine-13 (Ile13), located in the clamp loop that undergoes conformational changes under shear force exerted on the bacteria upon binding to its receptor (Figure 1). Molecular dynamic simulations confirmed that a subpopulation of the C-mannoside conformers was able to interact in this secondary binding site of FimH, thus unraveling a new mode of binding, useful in the design of potent inhibitors against the E. coli adhesion.

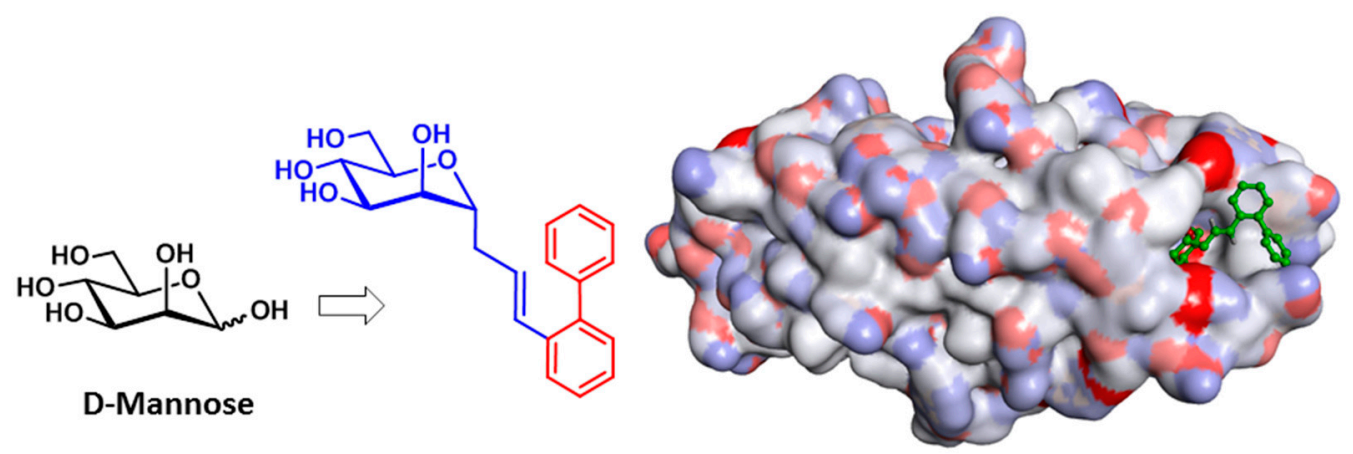

Figure 1. Docking of ortho-substituted phenyl ring to the FimH adhesin of E. coli.

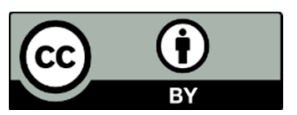

(C) 2019 by the authors. Submitted for possible open access publication under the terms and conditions of the Creative Commons Attribution (CC BY) license (http://creativecommons.org/licenses/by/4.0/). 